\title{
YACIMIENTO DE DIATOMITA EN TACNA
}

Gualberto Tejada Bedoya ${ }^{I}$

\author{
$R E S U M E N$
}

El yacimiento de diatomita se encuentra ubicado en el altiplano del departamento de Tacna.

El objetivo del proyecto es aprovechar un recurso natural mineral denominado diatomita que, mediante la extracción, transporte y procesamiento industrial, mejora sus condiciones naturales para que pueda ser usado como filtro-ayuda y en una infinidad de usos industriales.

\section{A B STRACT}

The mining place of diatiomita is located in the highlands of the department of Tacna.

The objetive of this project is to take advantaye of the natural mineral resource named diatomita, which througlr its extraction, transport and industrial process, improve i'ts natural conditions in order to be used as helping-filter and in a wide industrial uses.

\section{INTRODUCCION}

Pocos minerales juegan un rol tan importante en la vida actual de la humanidad, tal como la diatomita o tierra de diatomeas, el cual es un producto mineral compuesto esencialmente de sílice, resultante de la fosilización de organismos fitoplanctónicos microscópicos.

En realidad, mucho del agua y bebida que tomamos o alimentos que comemos han sido purificados y/o clasificados en alguna etapa del proceso de su producción por medios de la diatomita, y esto debido a la casi única combinación de propiedades físicas y químicas.
Los usos principales de la diatomita son:

- Como filtro-ayuda para: cerveza, melaza, aceites, vinos, antibióticos y otros productos alimenticios.

- Como relleno en la fabricación de papel, jebe sintético, pinturas, plásticos moldeados, etc.

- Como aislante de temperatura y sonido en estructuras, como materia prima en la fabricación de ladrillos, bloques, accesorios eléctricos, como abrasivos para la limpieza y pulidor de metales.

En el altiplano del departamento de Tacna existe un gran yacimiento que puede ser aprovechado para atender necesidades locales, nacionales e internacionales. 
No existe en el país una planta de procesamiento de diatomita para filtro-ayuda, la diatomita es importada de México y de Estados Unidos.

Tacna tiene una ubicación estratégica en el continente sudamericano que permitirá una fácil comunicación con Bolivia, Argentina y Brasil a través de la carretera Trans-Amazónica y la carretera binacional llo Desaguadero-la Paz, asimismo, a través del Puerto de llo y de Matarani a diferentes partes del mundo.

\section{MARCO TEÓRICO}

Actualmente el proyecto tiene asegurado el denuncio minero con reservas superiores a los cinco millones de toneladas.

\section{GEOLOGÍA LOCAL}

Estos depósitos están constituidos por acumulaciones arcillo-arenosas, limos y delgados lechos de conglomerados finos que permiten estratificaciones en lechos muy delgados.

El depósito en estudio es de origen lacustre debido a que las diatomitas estudiadas no son marinas. El lago fue poco profundo y de aguas limpias, con condiciones ecológicas favorables para la proliferación de diatomitas. El depósito de diatomita tiene una cubierta de material fluvial con espesores que varian de $0,20 \mathrm{~cm}$ a $4.00 \mathrm{~m}$

\section{ESTUDIO MICROPALEONTOLÓGICO}

La muestra presenta un color blanco, es de granulometría fina, tiene un brilio mate terroso, reacciona con el ácido clorhídrico.

Se ha observado abundancia de frústulas de diatomeas:

$\begin{array}{ll}\text { Enteras } & 50 \% \\ \text { Fraccionadas } & 20 \% \\ \text { Otros elementos } & 30 \%\end{array}$

\section{ANÁLISIS DIATOMOLÓGICO}

Las especies de diatomeas observadas presentan frústulas de forma: alargada, lanceolada, elíptica y fusiforme.
Las frústulas rotas generalmente corresponden a las formas grandes. Estas diatomeas pertenecen al orden de Pennales, entre los géneros que se encuentran tenemos: Fragilaria, Cymbella, Comphonema, Anomaeoneis, Suririlla.

El tamaño de toda diatomita es un organismo vivo denominado diatomea. Este organismo es una planta sin florescencias.

En todos sus géneros y especies, tiene la capacidad de extraer sílice de su habitat acuoso, con dichas extracciones forma una concha que consiste principalmente de Silice amorfa precipitada biológicamente.

\section{ANÁLSIS DE LA DEMANDA POTENCIAL}

Mercado Interno. Un $70 \%$ de la demanda de diatomita en el Perú está orientado hacia los filtroayuda. En este campo son dos las industrias que destacan considerablemente:

- Industria Cervecera

- Industria Azucarera

Mercado Externo. Con el presente estudio calculamos el Mercado Potencial de Diatomita Procesada Peruana en el Grupo Andino. Se calcula en forma preliminar el mencionado potencial planteando el impuesto que las exportaciones peruanas capturan un $80 \%$ de las importaciones de los paises GRAN. Venezuela es el mayor importador con un promedio de $50 \%$ el segundo importador es el Perú con un promedio de $24 \%$, Colombia se constituye en un tercer importador con $13 \%$ seguido de Ecuador y Bolivia con $4 \%$ y $3 \%$ respectivamente.

\section{ASPECTOS GENERALES}

\section{1) Ubicación Geográfica}

El yacimiento de diatomita del presente estudio se encuentra ubicado en:

\section{Paraje}

Jalaropampa y Alcocollopampa (Zona Tripartito frontera Perú, Bolivia y Chile)

$\begin{array}{lll}\text { Distrito } & : & \text { Palca } \\ \text { Provincia } & : & \text { Tacna }\end{array}$


Departamento

Tacna

Altura

4110 - 4160 m.s.n.m

\section{Coordenadas UTM}

\begin{tabular}{|c|c|c|}
\hline PUNTO & NORTE & ESTE \\
\hline VNE & 8066100 & 450000 \\
\hline VSE & 8065300 & 450000 \\
\hline VNO & 8064300 & 446700 \\
\hline VSO & 8063700 & 446700 \\
\hline
\end{tabular}

\section{2) ACCESIBILIDAD}

El acceso al yacimiento es por la carretera Tacna Collpa - La Paz, presentando las siguientes caracteristicas:

\begin{tabular}{|c|c|c|c|}
\hline DESDE & HASTA & $\begin{array}{l}\text { DISTANCIA } \\
\text { (Km) }\end{array}$ & $\begin{array}{c}\text { TPO DE } \\
\text { CARRETERA }\end{array}$ \\
\hline $\begin{array}{l}\text { Tacna } \\
\text { Km } 41+800 \\
\text { Palca } \\
\text { Alto Perú } \\
\text { lom } 145+\infty 00\end{array}$ & $\begin{array}{l}\mathrm{km} 41+800 \\
\text { Palca } \\
\text { Alto Perú } \\
\mathrm{km} 145+000 \\
\text { (desví) } \\
\text { Tripartito }\end{array}$ & $\begin{array}{c}41,8 \\
10,2 \\
80 \\
13 \\
\\
13\end{array}$ & $\begin{array}{l}\text { Asfaltada } \\
\text { Afimada } \\
\text { Afimada } \\
\text { Afimada } \\
\text { Afimada }\end{array}$ \\
\hline
\end{tabular}

\section{ESTUDIO DE MERCADO}

\section{Descripción y aplicación del producto}

El producto podrá ser ofertado en tres formas, clasificadas de acuerdo con su proceso.

\section{- Diatomita natural}

Se logra mediante un chancado primario, seguido por una molienda y secado simultáneo, con una corriente de gases calientes. Las partículas suspendidas son arrastradas y pasadas por una serie de ventiladores, ciclones, separadores y filtros, obteniéndose, como resultado, la separación de polvo de distintos tamaños, la remoción de residuos de impurezas y la expulsión de agua absorbida, obteniéndose un producto uniforme, para brindar mayor claridad en el líquido filtrado pero menos velocidad de flujo. Su color en blanco. Este producto, sin otro proceso, puede ser embolsado o manejado a granel como producto natural.

\section{- Diatomita calcinada}

Se le da el mismo proceso anterior, pero se hace un ajuste de tamaño de partícula, calcinando el material en polvo en hornos rotatorios, seguidos de una molienda y clasificación, resultando un producto con tinte rosado debido a la oxidación del óxido de hierro contenido.

\section{- Diatomita calcinada con fundente}

Se da el mismo proceso preliminar (natural), pero se le agrega un fundente (carbonato de sodio, cloruro de sodio), que elimina el tinte rosado por la formación de un complejo de silicatos de sodio, aluminio y hierro, obteniéndose un producto blanco de buena calidad, aumenta el efecto relacionado con el tamaño de la partícula y características de superficie $y$, consecuentemente, un material más permeable, luego es clasificado neumáticamente, separando el producto en grados de diferente tamaño.

Existe una gran aplicabilidad industrial de la diatomita. Para nuestro proyecto lo resumimos asi:

- Aplicaciones principales.

- Aplicaciones secundarias.

Las aplicaciones principales son las que presentan una mayor utilización en la industria, un mayor empleo en cuanto a volumen y para cuyos usos la diatomita es más conocida.

Dentro de esta clasificación y para efectos del estudio de investigación, se utiliza:

Como filtro-ayuda en la fabricación de cerveza, gaseosas y aguas de mesa, vino, aceite y refinado de azúcar.

Como carga o relleno funcional (pinturas, esmaltes, barnices) en la industria de caucho e industria de plástico.

Como absorbente en el control de resinas en la fabricación de papel, cartulina y cartón.

Las aplicaciones secundarias son aquellas que son menos conocidas; su empleo se realiza generalmente en pequeña escala, para efectos dei 
estudio de mercado no tendrán incidencia, pero existen suficientes expectativas para un futuro promisor. Se pueden nombrar los siguientes usos:

Filtración, clarificación de jugos, industria farmacéutica, fabricación de productos químicos orgánicos e inorgánicos, depuración de aguas industriales, municipales y domésticas, producción de margarina, aceites minerales, bebidas alcohólicas.

Relleno funcional, en insecticidas, fertilizantes. Abrasivo suave (abrillantadores de plata y automóviles). Aislante térmico y acústico, en forma de polvo o de ladrillos naturales calcinados, fabricación de aisladores de alta temperatura.

Recubrimiento de estanques y tuberias de lixiviación del cobre, gracias a su resistencia al ataque de soluciones ácidas.

Como desmoldante en procesos metalúrgicos de fundición que exigen materiales con elevado punto de fusión, resistencia al peso y al desgaste provocado por materiales fundidos.

Como filtrante en la industria del petróleo, el mercado potencial y futuro de los países productores de la Comunidad Andina(CAN) tiene una gran expectativa.

En la actualidad, se van abriendo nuevos mercados para este producto, lo mismo ocurre con las aplicaciones futuras de los productos diatomáceos. El oligopolio que ejercen grandes compañías instaladas en EE.UU., países de la exURSS, Dinamarca, Alemania, México, España, entre los de mayor producción, en donde un factor que no se puede evaluar o catalogar en la tendencia de crecimiento es la influencia enorme que han ejercido los departamentos de investigaciones, buscando nuevas aplicaciones para sus productos.

La investigación y el desarrollo definitivo, en la Universidad de Nevada y el Ministerio de Energía de Ios EE.UU., para el uso de la diatomita en sitios contaminados nucleares existentes en los EE.UU. Fuente: Landmark - USA.

Actúa como enmienda del suelo y acondiciona el área de aterrizaje (Play Ball), Eagle . Picher i. b.p. USA.

Se usa para filtrar partículas clasificadas como metales pesados, orgánicos y materiales radiactivos tales como Cesio, Estroncio y Tritio, asociados comúnmente a las instalaciones nucleares. Fuente: Landmark A. C. T. - USA.
Producción de polietileno, se pronostica su crecimiento en todo el mundo en un índice de 5 - 7 $\%$ por año, trayendo demanda de diatomita.

Fuente: Diatoms econom. 7a ed. USA.

Producción de plumas estilográficas de porcelana con una arcilla artificial de diatomita purificada, cristaleria coloreada de diatomita.

Fuente: Industrial Research Institute of Ishikawa - Japón.

Síntesis de Wollanstonita, tratando una mezcla de una composición formada por una diatomita española y una creta y diversos mineralizadores.

Fuente: Instituto de Cerámica y Vidrio, Departamento de Cerámica C.S. I.C. Arganda del Rey (Madrid) Espaza .

Muchos materiales (perlita, celulosa, carbón) vienen siendo sustituidos, y viceversa, por los productos diatomáceos por ser un material superior; sin embargo, aún se mantienen por tener precios menores que fácilmente podrían ser desplazados mediante precios competitivos.

Las siguientes son especificaciones y test usados comúnmente:

\section{- ASTM:}

D 604 - 42 Pigmentos de sílice diatomácea.

D 719 - 63 Análisis de pigmentos de sílice diatomácea.

C - 517 - 71 Tierras diatomáceas como aislantes.

\section{- NORMA MILITAR:}

MIL - S - 15191 B Sílice diatomácea(pigmentos)

MIL - S - 20550 B Tierras diatomáceas

MIL - F - 52637 Ayuda-filtrante, purificación de agua.

S2-MA-522 QPigmento de sílice diatomácea

\section{- ESPECIFICACIONES TAPPI}

T - 658 - 0S - 77 Propiedades de silice diatomácea. 


\section{2. Área geográfica del mercado}

Para seleccionar el mercado de la producción del proyecto, se debe tener en cuenta lo siguiente:

- La experiencia que se tiene en los mercados en que se conocen los productos.

- La ubicación geográfica de la provincia de Tacna dentro de los países de la Comunidad Andina.

- El costo del transporte tiene una relación directa con la distancia; la ubicación de Tacna permite aprovechar ventajosamente esta variable, en relación con la variable precios, que puede manejar el oligopolio de productos extranjeros, pues por muy bajo que sea el precio de la competencia, este siempre estará sujeto al incremento por distancia y transporte.

Consideración de pocos męrgados al inicio de la producción.

- La calidad del producto que permitirá desplazar a la competencia.

- El estudio se clasifica de acuerdo con la extensión geográfica.

- Por lo expuesto, teniendo en cuenta su mayor accesibilidad y viabilidad, se pueden sugerir los siguientes mercados:

\begin{tabular}{|l|l|}
\hline \multicolumn{1}{|c|}{ MERCADOS } \\
POTENCIALES & \multicolumn{1}{|c|}{ MERCADOS } \\
& COMPLEMENTARIOS \\
\hline - Territorio Nacional & - Colombia \\
- Brasil & - Chile \\
- Argentina & - Venezuela \\
- Bolivia & - Uruguay \\
- Ecuador & - Paraguay \\
\hline
\end{tabular}

\section{GEOLOGIAA REGIONAL}

\section{Unidades Estratigráficas}

Se han encontrado afloramientos de rocas volcánicas y sedimentarias con edades comprendidas entre el terciario medio y el cuaternario reciente.

\section{Terciario}

BASAMENTO, formado por la intercalación de agiomerados andesíticos, riolíticos, dacíticos y rocas sedimentarias, aflorando en el cerro Pacocahua, como consecuencia de un levantamiento tectónico en forma de domo. En el cerro Sirque se aprecian unas capas volcánicas de coloración verde y blanquecina, cerca a la frontera con Chile se pueden ver unos conglomerados de fragmentos hasta de 20 $\mathrm{cm}$ de diámetro con una potencia de $20 \mathrm{~m}$, que pertenecerian al tope de este basamento o la base de la formación Maure.

FORMACIÓN MAURE, consiste en una intercalación de conglomerados volcánicos, limolitas, areniscas, tufos retrabajados y brechas volcánicas, la coloración es variada: amarilienta, verde, gris, blanquecina y pardusca.

VOLCÁNICO SENCCA, constituido por tufos riolíticos y dacíticos, existen afloramientos al norte y noreste del Ayro, se le asigna una edad entre el Plioceno Medio y Superior.

FORMACIÓN CAPILLUNE, consiste en una intercalación de arcillas, limolitas, areniscas, conglomerados y tufos redepositados. Aflora restringidamente en la quebrada del río Uchusuma por el sector del campamento del Ayro y la presa Paucarani. Su edad: el Plioceno Superior.

VOLCÁNICO BARROSO, consiste en una secuencia de rocas volcánicas cuyos afloramientos forman conos, muchos de ellos irregulares, constituyendo las montañas. Su edad asignada es del Plio-Pleistoceno.

\section{Cuaternario}

Estos depósitos se extienden en casi la totalidad de las pampas del Ayro.

\section{DEPÓSITOS MORRÉNICOS $Y$} FLUVIOGLACIARES, constituyen las morrenas terminales y laterales de antiguos glaciares que formaron los valles que hoy subsisten. Se encuentran principalmente en las partes altas y laderas de los cerros Queñuta, Condorpico, Nevado Huancane.

Los depósitos fluvioglaciares se encuentran en los lugares bajos inmediatos a las laderas y morrenas consisten en una mezcla de arena y grava gruesa con abundante matriz limo-arenosa, de color pardo, friable, estratificación grosera.

DEPÓSITOS RECIENTES, coluviales que son acumulaciones de materiales provenientes de desmoronamientos y deslizamientos, mezclados $\sin$ clasificación entre bloques angulosos, heterogéneos 
y finos, presentados baja consolidación.

Fluviales, constituidos por gravas poligénicas de forma subangular a subredondeada y arenas gruesas, que se encuentran rellenando los lechos de los ríos y quebradas.

\section{GEOLOGÍA LOCAL}

\section{Depósitos lacustres}

Están constituidos por acumulaciones arcilioarenosas, limos, delgados lechos de conglomerados finos y un desarrollo notable de diatomeas que proliferaron en un ambiente lacustre somero de aguas tranquilas muy ricas en sílice. El lago fue poco profundo, de aguas limpias, con condiciones ecológicas favorables para el crecimiento de las diatomeas.

El depósito tiene una sobrecarga de material cuaternario que varía desde $0,20 \mathrm{~cm}$ hasta $3,00 \mathrm{~m}$, éste último espesor para el lado de la frontera con Chile. El yacimiento presenta una potencia máxima aproximada de $8-6 \mathrm{~m}$.

\section{Génesis del yacimiento}

El yacimiento tuvo su origen en la acumulación de las caparazones siliceas o frústulas de las diatomeas, las cuales al finalizar su ciclo vital caen al fondo de la cuenca, formando mantos de diverso espesor, con pequeñas intercalaciones de limolitas y arenas, constituyendo un típico recurso de origen sedimentario biogénico.

Las condiciones ambientales más favorables para el crecimiento de las diatomeas y, por ende, para la generación de depósitos de diatomita de interés comercial, son las siguientes:

Cuencas amplias y poco profundas, de modo que pueda ocurrir la fotosíntesis.

Abundante y continuo aporte de sílice soluble. La sílice normalmente proviene de períodos de intensa actividad volcánica cercana a la cuenca, lo cual restringe la vida y desarrollo más importante de las diatomeas principalmente al Terciario Superior Cuaternario.

Abundante aporte de nutrientes.

Ausencia de materiales tóxicos.
Ausencia o aporte mínimo de material clástico.

El desarrollo del fitoplanckton está determinado por las corrientes frías de surgencia, las cuales aportan los principales nutrientes de los vegetales: nitrógeno, fósforo y oxígeno, desde las profundidades de la cuenca. Al llegar a la zona de la fotosíntesis, favorecen una intensa floración de vida orgánica como fenómeno bioquímico.

Existiendo las condiciones para su desarrollo y luego de su muerte y depositación en el fondo, se generan los depósitos comerciales de diatomita, integrados tanto por los caparazones como por el fango diatomáceo.

Debido a la naturaleza de las frústulas, los depósitos de diatomita de interés industrial no deben haber sufrido los efectos de metamorfismo regional o de alteración química.

El yacimiento de diatomita de la zona del tripartito cumplió todas las condiciones ambientales y cumple la naturaleza de sedimentación de las diatomitas comerciales.

\section{GEOLOGÍA ECONÓMICA}

\section{Generalidades}

A través del proyecto desarrollado en el yacimiento de diatomita de JalaropampaAlcocollopampa (zona del Tripartito) y sobre la base de estudios previos, la sociedad minera denunció 1000 Há, efectivizándose en una primera etapa el levantamiento topográfico de 99 Há., mapeo geológico y cortes transversales para el cálculo de reservas También se proyectó la apertura de 55 pozos distribuidos en la totalidad del denuncio, efectuándose 20 pozos y algunas calicatas.

En una segunda etapa se realizaron el levantamiento topográfico de 158 hectáreas, mapeo geológico y cortes transversales para calcular las reservas. En ambas etapas se realizó el muestreo necesario y se envió a laboratorios, para el análisis físico-quimico del mineral y así determinar sus características y calidad que serán expuestas más adelante.

Los grosores de acumulación de diatomita son similares en todo el yacimiento, que van desde 3 a 8 $\mathrm{m}$. La sobrecarga de material aluvial varía desde 
$0,20 \mathrm{~m}$ hasta $3,00 \mathrm{~m}$ aproximadamente, teniendo sus valores más bajos hacia el Nor-Oeste y los más altos paralelamente hacia la frontera con Chile.

\section{CARACTERISTICAS FISICO-QUÍMICAS DEL MINERAL}

\section{- ESTUdIO FÍSICO-QUIMICO}

$\begin{array}{ll}\text { Densidad en banco } & 0,70 \mathrm{t} / \mathrm{m}^{3} . \\ \text { Densidad suelta } & 0,49 \mathrm{t} / \mathrm{m}^{3} . \\ \mathrm{PH} & 8,42 \\ \text { Color } & \text { Blanco }\end{array}$

\section{- ELEMENTOS QUIMICOS (HECHO EN CANADÁ)}

$\begin{array}{ll}\mathrm{SiO}_{2} & 84,86 \\ \mathrm{Al}_{2} \mathrm{O}_{3} & 1,95 \\ \mathrm{Ca} \mathrm{O} & 1,29 \\ \mathrm{Mg} \mathrm{O} & 0,60 \\ \mathrm{Na}_{2} \mathrm{O} & 0,56 \\ \mathrm{~K}_{2} \mathrm{O} & 0,70 \\ \mathrm{Fe}_{2} \mathrm{O}_{3} & 1,38 \\ \mathrm{Mn} \mathrm{O} & 0,05 \\ \mathrm{Ti} \mathrm{O}_{2} & 0,15 \\ \mathrm{P}_{2} \mathrm{O}_{5} & 0,53 \\ \mathrm{Cr}_{2} \mathrm{O}_{3} & 0,01 \\ \mathrm{~L} \mathrm{Ol} & 7,93\end{array}$

CUADRO COMPARATIVO DE LOS PRINCIPALES YACIMIENTOS ESTUDIADOS EN EL PERU

\begin{tabular}{|c|c|c|c|c|c|c|c|c|}
\hline \multicolumn{9}{|c|}{$\begin{array}{c}\text { ANÁLISIS QUÍMICO DE LOS PRINCIPALES YACIMIENTOS } \\
\text { ESTUDIADOS EN EL PERÚ }\end{array}$} \\
\hline COMP. & PIU & $\begin{array}{l}\text { PUI } \\
2\end{array}$ & $\begin{array}{c}\text { PIU } \\
3\end{array}$ & PIU & $\begin{array}{c}\text { ICA } \\
5\end{array}$ & $\begin{array}{c}\text { ARE } \\
6\end{array}$ & AYA & $\begin{array}{c}\text { TAC } \\
8\end{array}$ \\
\hline $\mathrm{SiO}_{2} \%$ & 65,50 & 65,25 & 64,25 & 77,67 & 76,40 & $\varpi, 20$ & 79,00 & 84,86 \\
\hline $\mathrm{Al}_{2} \mathrm{O}_{3} \%$ & 2,04 & 2,77 & 5,34 & 3,42 & 6,80 & 5,20 & 7,37 & 1,95 \\
\hline $\mathrm{Fe}_{2} \mathrm{O}_{3} \%$ & 1,25 & 1,35 & 3,62 & 2,05 & 3,30 & 2,20 & 1,17 & 1,38 \\
\hline $\mathrm{CaO} O$ & 9,58 & 7,37 & 4,68 & 2,19 & 2,80 & 4,68 & 0,88 & 1,29 \\
\hline $\mathrm{Mg} O \%$ & 3,29 & 2,02 & 1,90 & 1,40 & 1,30 & 6,50 & 2,01 & 0,60 \\
\hline $\mathrm{Na}_{2} \mathrm{O} \%$ & 1,87 & 4,78 & 5,59 & 3,17 & N.D. & 1,18 & 0,85 & 0,56 \\
\hline $\mathrm{K}_{2} \mathrm{O} \%$ & 0,50 & 0,61 & 1,07 & 0,83 & N.D. & 1,10 & 0,62 & 0,70 \\
\hline $\mathrm{P} \times \mathrm{C} \%$ & 14,75 & 14,26 & 12,99 & 8,44 & 6,80 & N.D. & N.D. & 7,93 \\
\hline
\end{tabular}

1.Piura1- Bayóvar, Sondeo B63 (Área I), Muestra A1 (Verdeja et al.1990 b).

2.Piura 2-Bayóvar, Estación 58 (Área III), Muestra DC4 (Verdeja et al.1990 b).

3. Piura 3-Bayóvar, Escarpas de Virrilá:, Muestra VI ( (Verdeja et al 1990 b).

4.Piura 4 - Bayóvar, Cueva del Inca (Área III), Muestra Cl-D (Verdeja et al 1990 b).

5. Ica 5-Cerro Tiza, (ITINTEC 1978)

6. Arequipa 6-Tarucani, (Agramonte 1983) .

7. Ayacucho 7-Ayacucho, (Agramonte 1983)

8. Tacna 8-Tripartito, (Sociedad Minera María de las Nieves de Tacna)

PxC Pérdida por Calcinación.

\section{ANÁLISIS MICROPALEONTOLÓGICO}

Micropaleontológicamente, la diatomita del Tripartito presenta un rango porcentual de diatomeas presentes por campo al microscopio de aproximadamente $85 \%$.

El grado de conservación de las frústulas es elevado, alcanzando el $80 \%$ de enteras y el $20 \%$ fragmentadas.

Son predominantes las diatomeas del orden de los Penales, entre las cuales se encuentran: Annalus, Fragilaria, Amphora, Cymbella, Eunotia, Gomphonema 
Diatomea, Melosira, Pinnularia, Cyclotella, Synedra, Rhopalodia, Surirella, Nitzchia.

\section{ANÁLISIS FRACCIONOMÉTRICO}

Para determinar la granulometría del material se tamiza la muestra en seco, usando las mallas ASTM $200,270,325$ Y 400 , cuyos resultados fueron los siguientes:

\begin{tabular}{|c|c|c|}
\hline $\begin{array}{c}\text { FRACCIÓN MAUAS } \\
\text { ASTM }\end{array}$ & $\begin{array}{c}\% \\
\text { EN PESO }\end{array}$ & $\begin{array}{c}\text { TAMAÑO DE PARTAULAS } \\
\text { (Mcras) }\end{array}$ \\
\hline+200 & 2,73 & +74 \\
+270 & 5,00 & +53 \\
+325 & 7,20 & +44 \\
+400 & 6,80 & +37 \\
-400 & 78,20 & -37 \\
\hline
\end{tabular}

\section{ELECCIÓN DEL MÉTODO DE EXPLOTACIÓN}

Para elegir el método de explotación se deben analizar diversas características y condiciones, con el objeto de minimizar los costos de explotación y optimizar la producción.

\section{CONDICIONES NATURALES DEL. YACIMIENTO}

- El yacimiento es de origen sedimentario, lacustre.

- Disposición casi horizontal del yacimiento y poca sobrecarga

- El yacimiento carece completamente de estratificación y se presenta como una masa homogénea suave.

\section{CONCLUSIONES}

1. En el departamento de Tacna se dispone de yacimientos de diatomita de buena calidad y de gran magnitud, garantizando la factibilidad del proyecto. La evaluación del yacimiento ha permitido comprobar reservas aproximadas a $5082647 \mathrm{t}$ (deducido el $30 \%$ de ajuste).

2. El potencial económico del yacimiento de diatomita del Tripartito ofrece buenas expectativas, exexpectativas,que permitirán una explotación extensiva aproximada de 164 años, de acuerdo al tamaño del proyecto (tomando en cuenta la máxima capacidad, correspondiente a 30845 t/año).
3. La demanda de diatomita como producto filtrante es amplia lo que se demostró en el estudio de mercado, teniendo la posibilidad en el país, de sustituir el uso de caolín en la industria del papel y otras por la diatomita como producto del relleno.6.

4. Los países de Sudamérica, por el proceso de desarrollo industrial se transforman paulatinamente en mercados potenciales para este producto.

5. El tamaño de la planta ha sido determinado en función comparativa de ingreso mas costo, existiendo entre una y otra alternativa una diferencia aproximada de $25 \%$ y el ingreso de ventas una diferencia de $50 \%$, pudiendo apreciar cual de las alternativas genera mas utilidades cuya capacidad máxima es de $21600 \mathrm{t}$.

6. La localización de la planta obedece al estudio de macrolocalización que determina feacientemente, su ubicación próxima al yacimiento o sea en el Tripartito, que según la evaluación cualitativa da como resultado ponderado de 1045 puntos frente a 820 puntos de la otra alternativa, y según la evaluación cuantitativa comparando el transporte del mineral mas el costo de terreno ofrece menor costo $\$ 851602,75$ frente a $\$ 3215091,30$ de la otra alternativa (Tacna).

7. La ubicación geográfica del yacimiento y la probable ubicación de la planta de proceso, otorga ventajas comparativas respecto a los productos norteamericanos.

8. Por las características naturales del yacimiento de origen lacustre sedimentario, en forma de capas horizontales, cercanas a la superficie y poco estéril, carente de estratificación y material homogéneo casi en todo el yacimiento, se ha elegido el método de explotación a cielo abierto y por canteras.

9. Las operaciones mineras se ejecutarán con equipo alquilado porque ofrece ventajas económicas.

10. Según la tecnología seleccionada para el proceso de producción, gran parte de la maquinaria y equipo es de fabricación nacional, disminuyendo los costos de flete y anulando impuestos de desaduanaje, sobre todo evita la fuga de divisas.

11. Las inversiones del proyecto serán financiadas con aporte propio y con crédito de la Corporación Financiera de Desarrollo, con la opción de aumentar el financiamiento si la empresa lo requiere. 
12. El precio de venta de los productos es a nivel de planta y asumiendo un $20 \%$ menos de precio que el mercado norteamericano, reflejando más atractiva su aceptación en el país y Sudamérica.

13. La implementación del proyecto permitirá el desarrolio de esa zona deprimida y de tanta importancia estratégica respecto a los países vecinos, así como redundará en la economía departamental y nacional respectivamente.

14. Se otorgará un valor agregado al mineral.

15. Se disminuirá la fuga de divisas por la sustitución de productos filtrantes y de rellenos importados.

16. Los indicadores de rentabilidad financiera son mayores a los económicos, lo que demuestra que el financiamiento adoptado es el correcto.

\section{REFERENCIAS BIBLIOGRÁFICAS}

DUNIN BORKOWSKI, E.: Minerales industriales del Perú-1996.

KADEY F, L.: "Diatomite ind. Min. and rocks skmol" pág. 87 91-1983.

LEMONS JIM F.: "Diatomite" - 1996.

LOPEZ JIMENO, C.: Estudio de vibilidad de proyectos.

ADUVIRE PATACA, O.: Mineros-1997.

PERRY JOHN, U.: Manual de ingeniería Quimica. Tomo 1 y 2 1968.

PETTIJER LEE.: Diatomité growth in the jece of adversity. Abril 1982

INSTITUTO DEINVESTIGACIÓN TECNOLÓGICA. : RecursosS no metálicos de Chile. 1995.

MANSILLA JOHNS.: Filtro ayudas. Pág. 47-69 diciembre 1981. MINERALES COMMODITY.: Diatomite. pág. 54,55,56-1996. 


\section{ANEXOS}
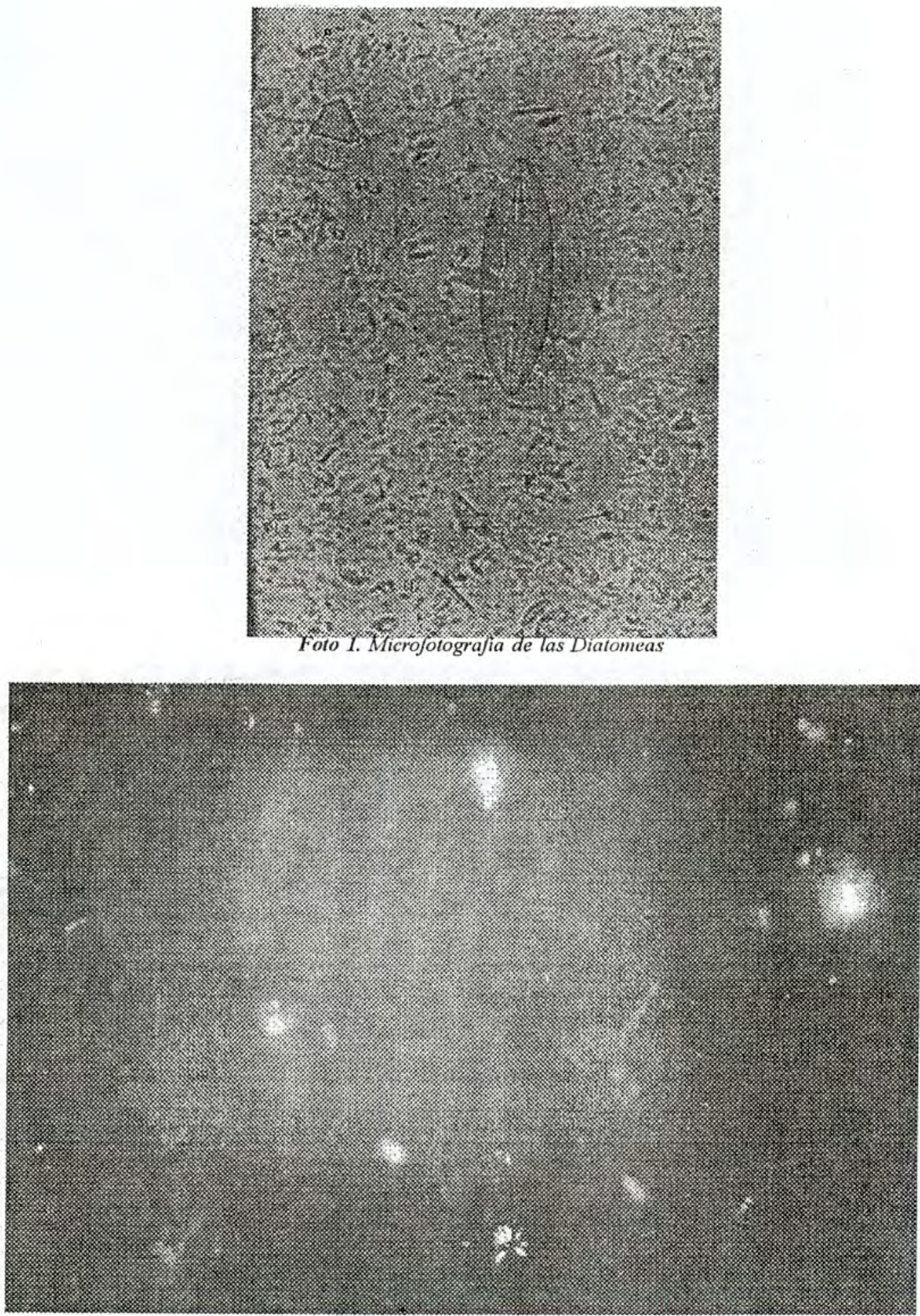

Foto 2. Microfotografia de la Asociación de diatomeas en nicolas cruzadas, se observan los componentes accsorios como calcita 


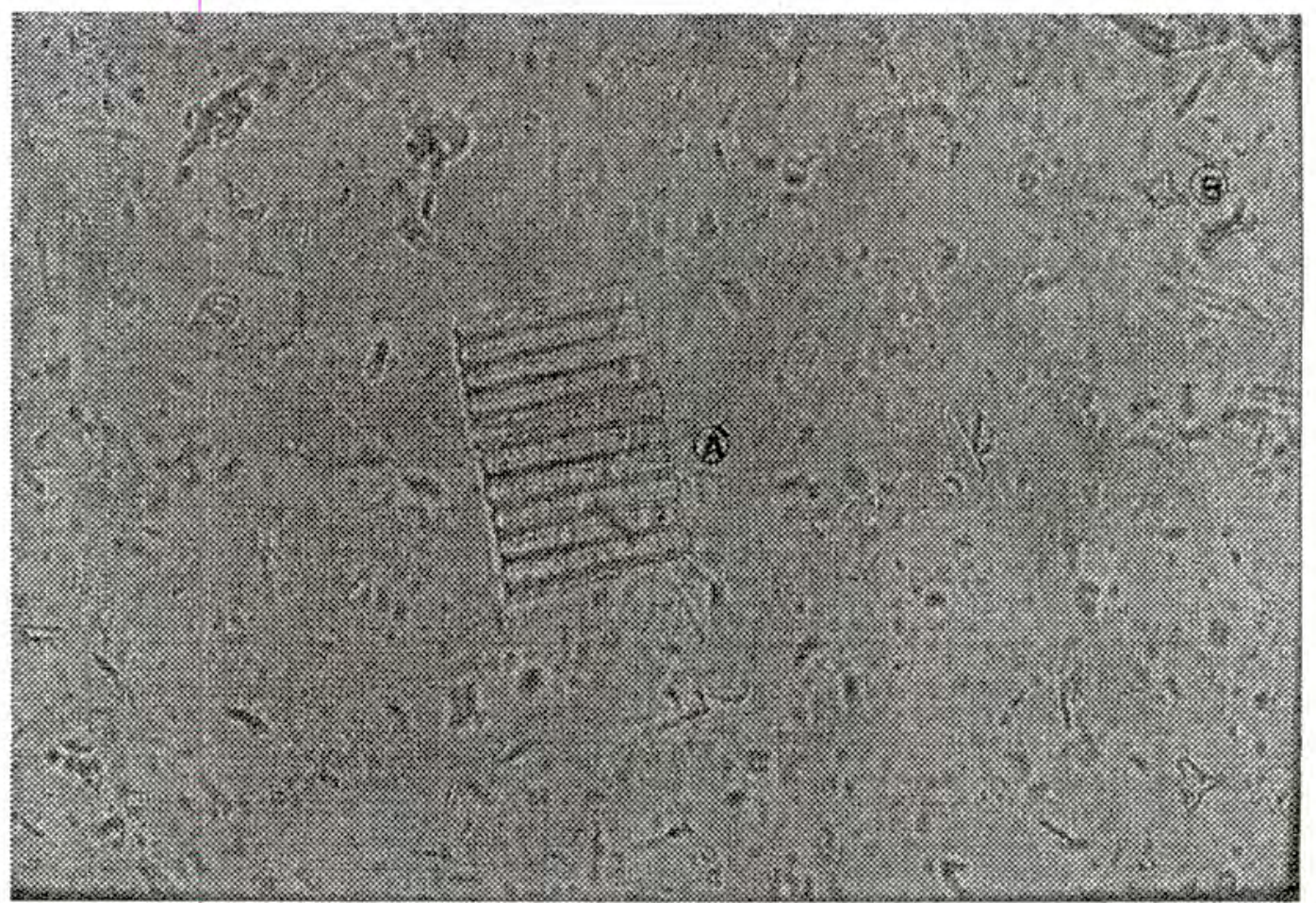

Foto 3. Microfotografias de las Diatomeas. Microfotografias de la asociación de diatomias aisladas, observe especies del género Fragilaria en cadenas (A) y soliarias (B).

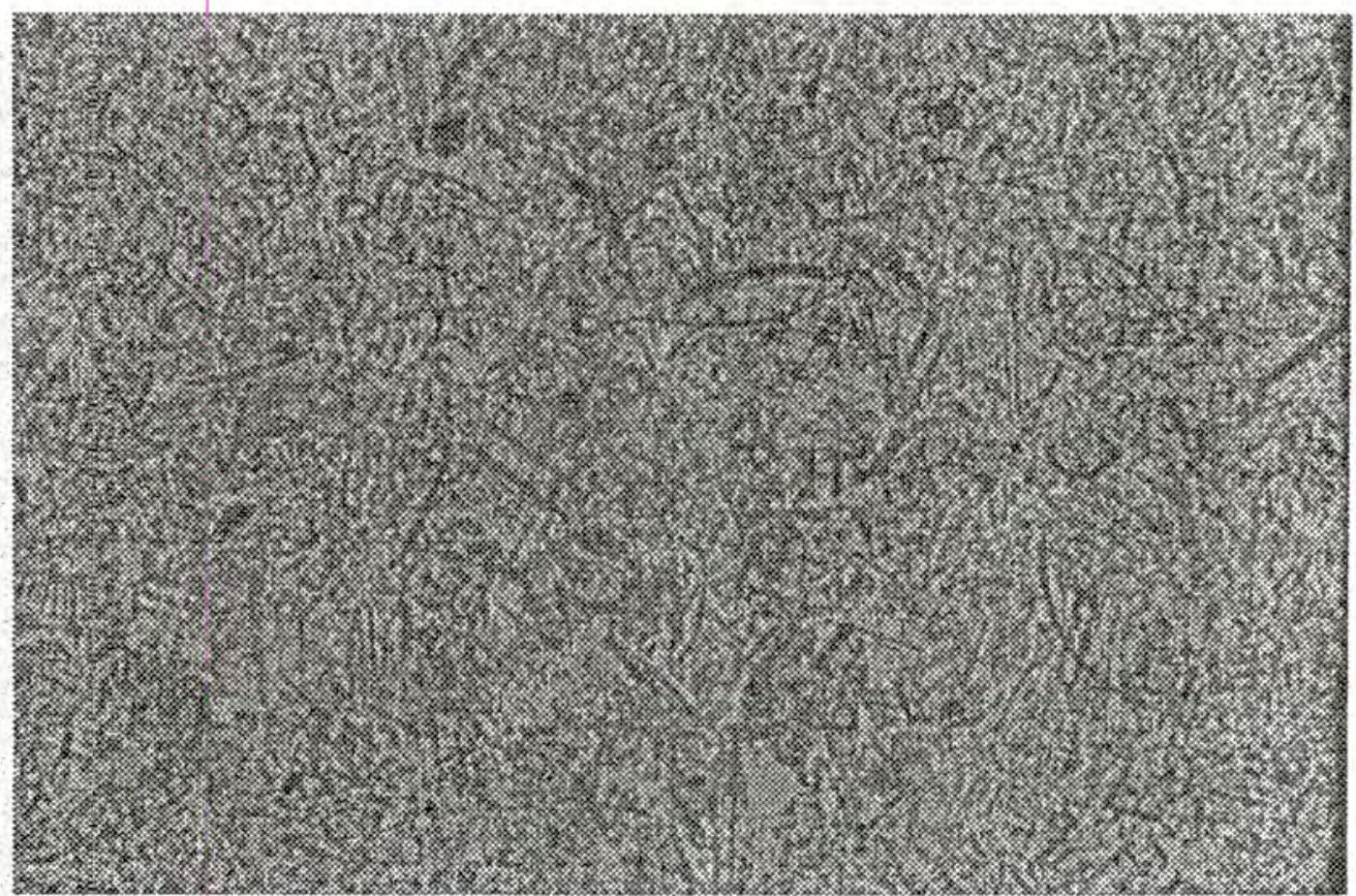

Foto 4. Microfolografia de la Asociación de diatomeas en la luz natural en un preparado concentrado $400 X$. 


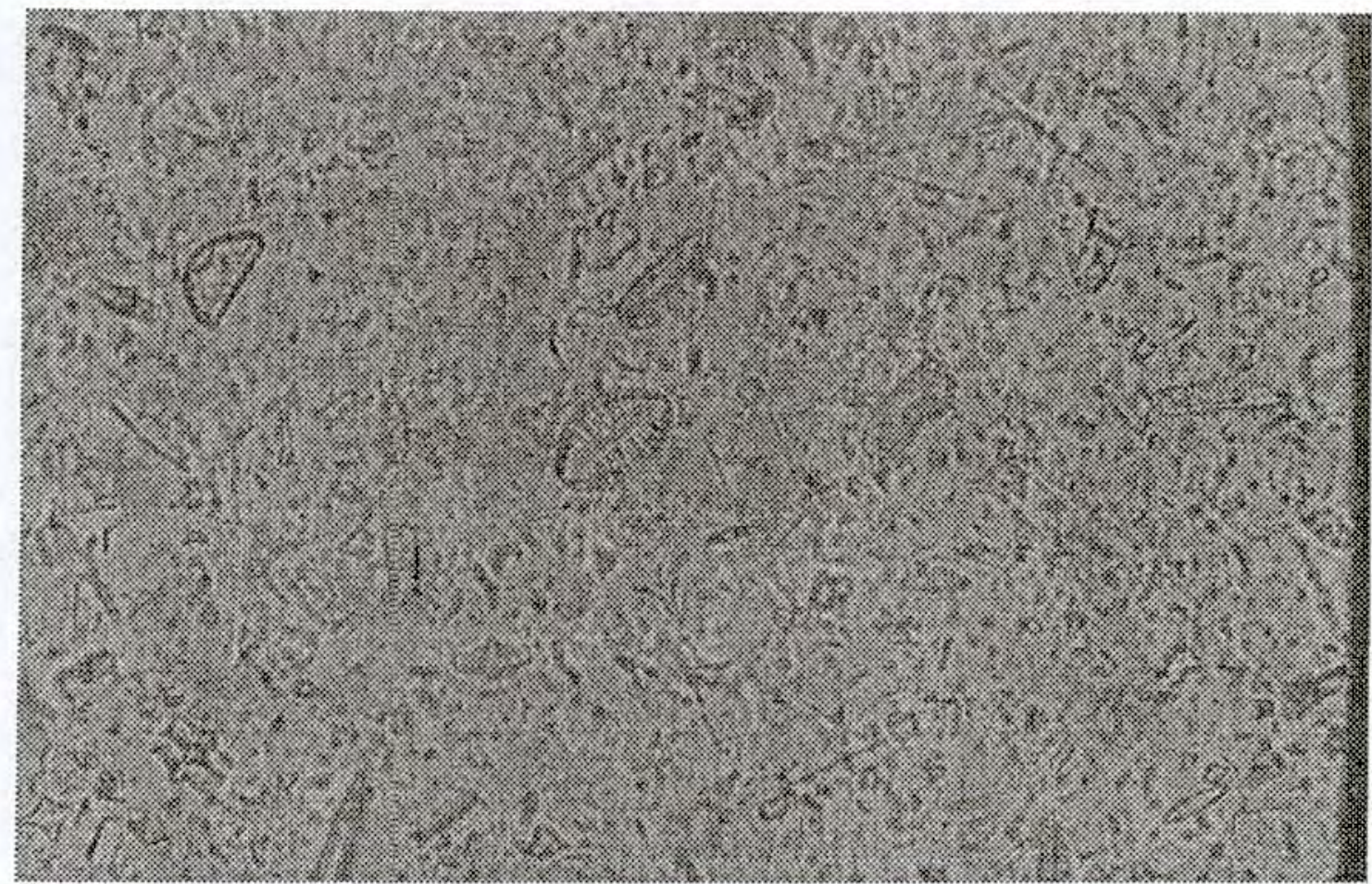

Foto 5. Microfotografias de la asociación de diatomeas en huz natural en un preparado menos concentrado $400 \mathrm{X}$.

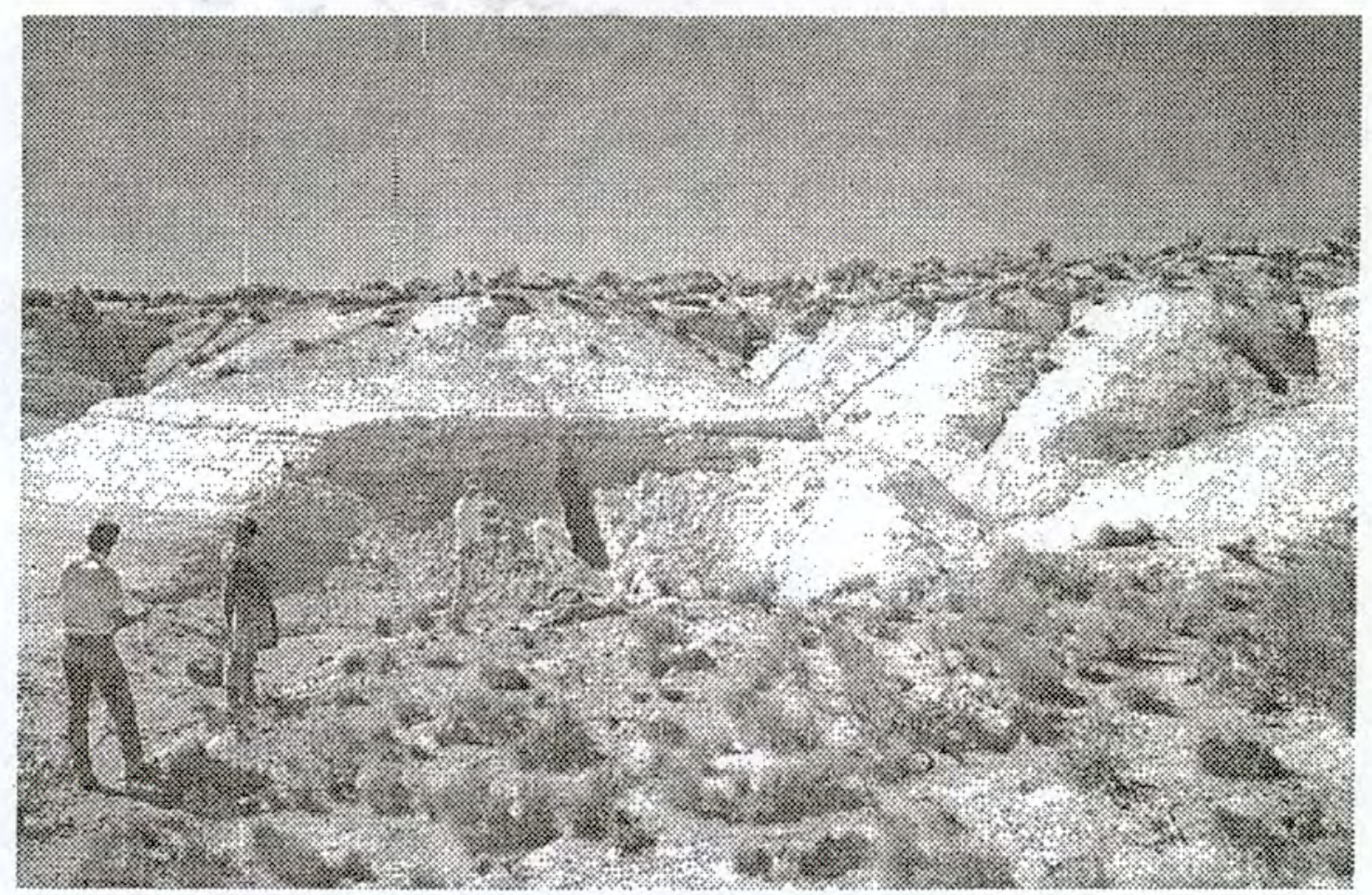

Foto 6. Yacimiento del Tripartito, mostrando los trabajos de muestreo geológico. 
Ciencia \& Desarrollo 7

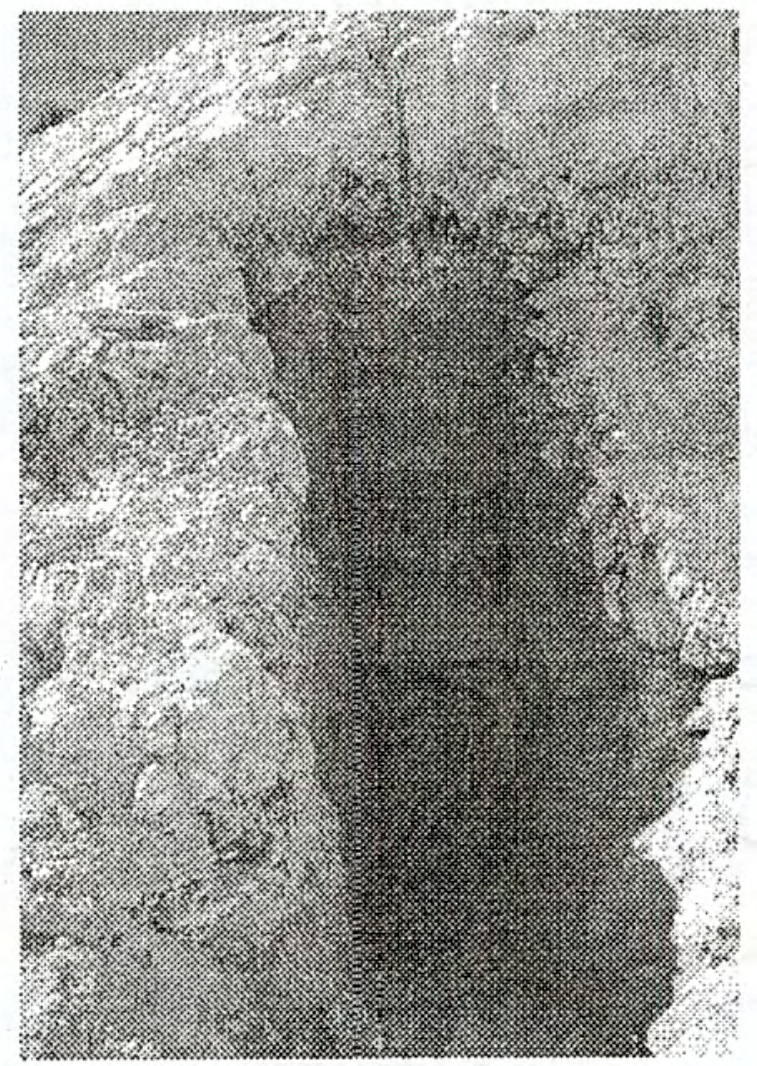

Foto 7. Vista de una calicata, se puede apreciar el registro geologico de su depositación. 


\section{CAIICATA E-12 COLUMNA LTTOLOGICA YAC. MARIA DE LAS NIEVES}

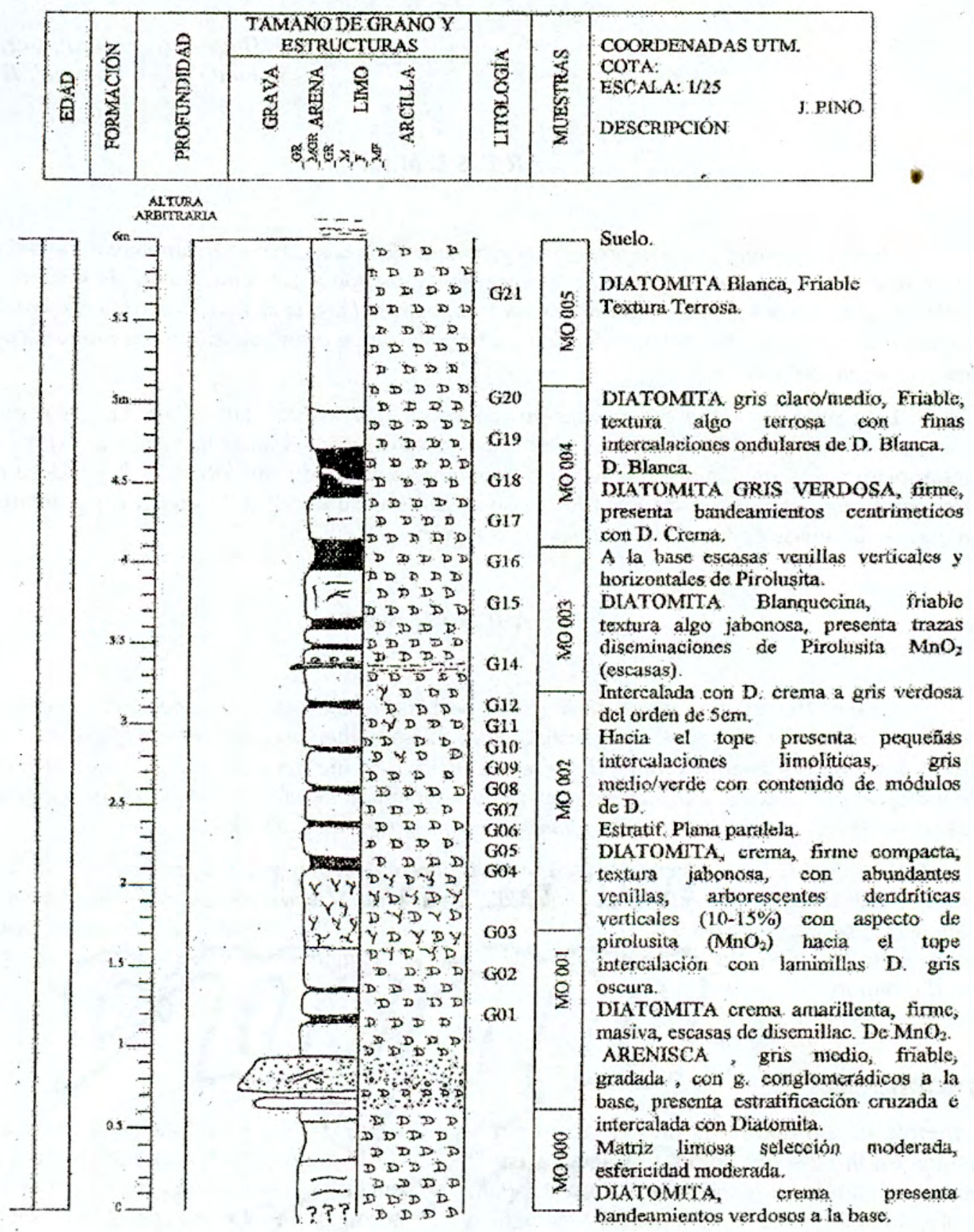

